\title{
A Survey Paper on Image Classification and Methods of Image Mining
}

\author{
Sandeep Pandey \\ Department of Computer Science and Engineering \\ MANIT, Bhopal, M.P., INDIA
}

\begin{abstract}
Image mining that consists of Image processing, Databases, Data mining, Machine learning, and artificial Intelligence, focuses on extraction of patterns from large collection of images. Although many researches have been done on many of these areas but issues are still there in image mining techniques. For instance, data mining techniques can't automate mining of images from large set of images. In this paper, a general approach is been discussed to mine data based on many researches done till now. It will help readers who want to know about existing image mining techniques, and knowledge mining from large image data set to progress in this area.
\end{abstract}

\section{General Terms}

Data mining, image processing, neural network, machine learning.

\section{Keywords}

Image mining, image classification, image clustering, image pre-processing, content based image retrieval (CBIR).

\section{INTRODUCTION}

Image mining is an interdisciplinary field that is based on Image processing, data mining, machine learning, databases and artificial intelligence. As the world has grown and social networking is growing dramatically, there is a need of extracting meaningful images out of the pool of network. The emphasis of image processing is on the understanding of certain characteristics of specific image and the focus of data mining is to apply algorithms to mine data (images) more accurately and efficiently. On the other hand, the prominence of machine learning is to make network learn about patterns/features so that errors in output can be minimized and to classify images using specific features selected during image processing. This paper briefly explains image mining process proceeded by literature reviews based on different papers, research gaps found, application of image mining techniques and lastly conclusion.

\section{IMAGE MINING PROCESS}

Image mining can be expressed as a sum of four essential phases (Figure 1), described as follows:

\subsection{Image Pre-Processing}

It is necessary to improve quality of image before using it to extract features form it. Images are pre-processed to create high quality images that result in more transparent categorization. Pre-processing of an image is also important for removing noise from the images that may cause unwanted results. There are linear and non-linear image filters to remove noise in the image. In pre-processing, we can do Image thresholding [1], edge detection [1], border tracing [1], wavelet based segmentation [2]. We may also use low pass filters, high pass and band pass filters [1] for removing noise from the images.

\author{
Sri Khetwat Saritha, PhD \\ Department of Computer Science and Engineering \\ MANIT Bhopal, M.P., INDIA
}

\subsection{Feature Extraction}

Features of an image play an important role in distinguishing and categorizing set of images in their respective categories. There are many feature like color, edge, texture, shape, and boundary that can be extracted from the image. There are various method used for feature extraction described next.

\subsubsection{Image Thresholding}

It is the simplest method of image segmentation. Extreme contrast stretching results thresholding. From a gray-scale image, thresholding can be used to create binary images. In simple way, it is a process of replacing all image pixels $\mathrm{Ii}, \mathrm{j}$ (indicates intensity values of pixel located in $\mathrm{i}^{\text {th }}$ row and $\mathrm{j}^{\text {th }}$ column), whose intensity is less than a fixed or constant intensity, called thresholding of an image.

\subsubsection{Edge detection}

Variation of features of scene like brightness, gives rise to edges. These are representations of discontinuities of intensity function of an image. The purpose of detecting sharp discontinuities in image brightness is to capture important and useful changes in properties of the world. It can be shown that under general assumptions for an image, discontinuities in image brightness corresponds to discontinuity in depth, discontinuities in surface orientation, change in material properties, and variations in scene illumination. There are various methods to detect these discontinuities as edges. All these methods are based on derivatives and can be classified into two major categories listed next.

\subsubsection{First Order Method}

Prewitts and Sobel operators [1] are some of the tool used for edge detection. These operators are $3 \mathrm{X} 3$ dimension masks that is to be applied on whole image to get edges.

\subsubsection{Second order method}

Laplacian operator [1] is used to get sharp edges in second derivative method. These are also called isotropic filters. It is very sensitive to noise. If an image contains noise, Laplacian will ruin the entire image.

\subsubsection{Boundary tracing}

It is also known as contour tracing of a binary digital region can be thought of as a segmentation technique that identifies the boundary pixels of a digital image. This is done tracing whole image pixels top to down, left to right and the result can be encoded in Run-Length [1] code.

\subsubsection{Image color extraction}

It can be used to extract color as a feature of the image. Color histogram feature in image is used to represent color distribution of the image. The red, green, blue distribution in an image can be controlled by using histogram equalization. In the process of equalization of an image, all red, green, blue pixels of that image are calculated, and redistributed to make image contrast balanced globally in the image. This allows the area of lower contrast to gain higher contrast. 


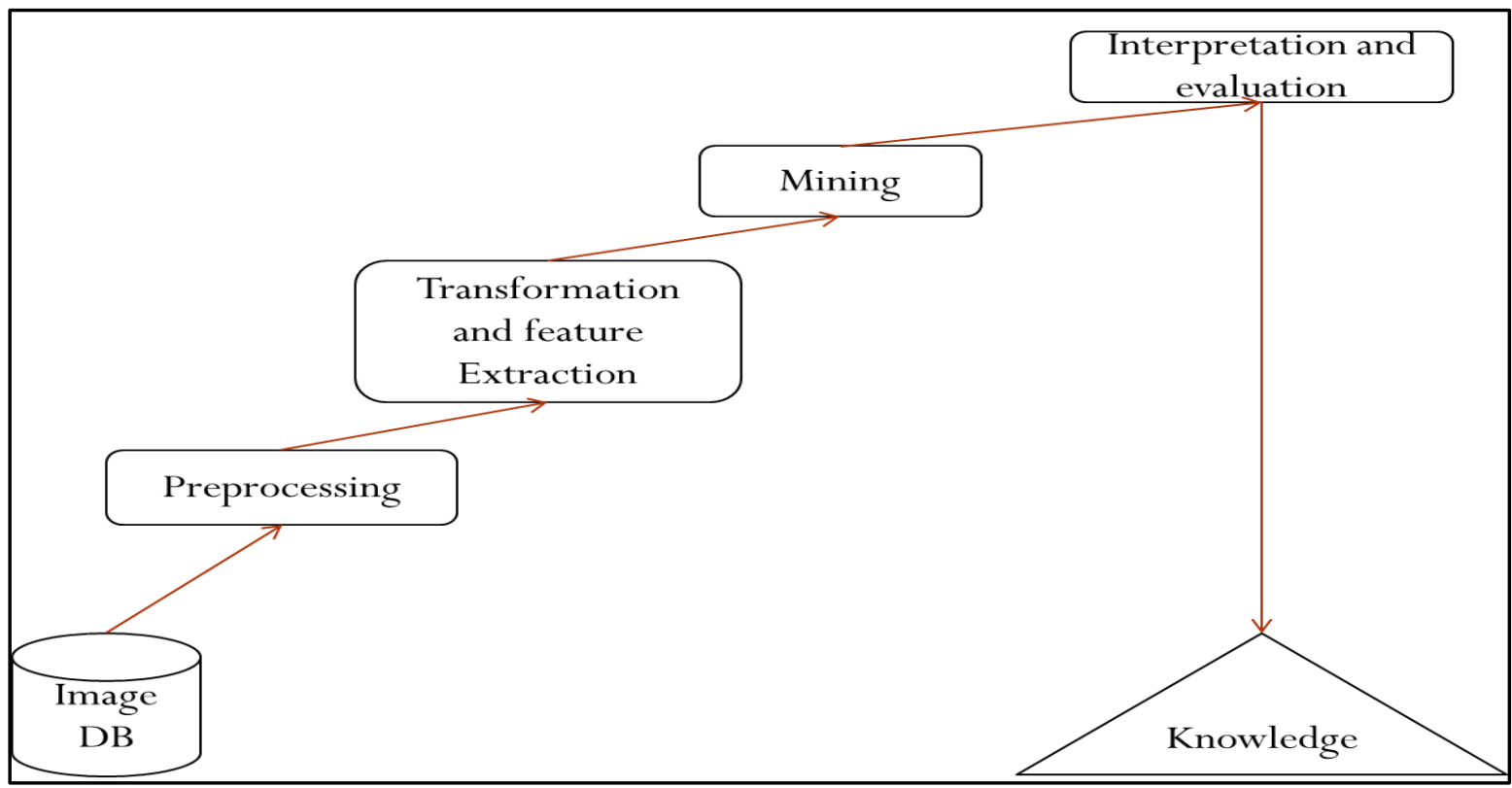

Figure 1: Image Mining Process [3]

\subsubsection{Image texture extraction}

Image texture gives us information about the spatial arrangement of color or intensities in an image or selected region of an image. It is set of metrics calculated in image processing which is used to quantify the texture of the image. Image textures are manually created or found in natural space.

\subsection{Image mining}

Mining useful knowledge from large set of image database is the main objective of image mining. Image mining is used in various forms based on queries (see Table 1) similarity search techniques (see Table 2) and type of learning such as supervised and unsupervised learning.

\subsubsection{Image classification [2]}

The intent of classification process is to categorize all pixels in a digital image into several classes. It is a supervised learning method used to classify images based on some preknown results. The main task here is to assign an input image one label from a fixed set of categories. All classification algorithms are based on the assumption that the image in question depicts one or more features and that each of these features belongs to one of several distinct and exclusive classes. Various classifier used for this purpose are- Bays classifier, Neural Networks (MLP, RBF, SVM and many more), Decision Tree classifier, Genetic Algorithms. Classification of image is done in mainly two phases, learning phase and test phase. In learning phase, images taken are different and learning is made on the basis of output class. In the testing phase, image features/specification are used to map image to an output class. One of the most important method in classification of image is decision tree. Based on whole sample, decision trees divide decision space to smaller areas as a return. Decision tree breakdown the complex problems into smaller problem based on some decision taken while taking action.

\subsubsection{Image clustering [4]}

It is an automated unsupervised method in which samples are divided in various groups based on some similar characteristics. These groups individually are called cluster. This means, cluster is a collection of objects, where object share some property/feature with some object in the cluster. There are various methods of clustering data in groups. Some of them are Partitioning methods, hierarchical methods, and Grid-based methods. Clustering don't require output feature vector like classification do, rather they continuously add new sample to a group based on their property that closely matches to any sample of the group and in this ways growing the size of cluster by adding more samples as they arrive.

\section{Table 1: Queries in Content Based Image Retrieval System}

\begin{tabular}{|c|c|}
\hline $\begin{array}{c}\text { Image Sample Based } \\
\text { Queries }\end{array}$ & $\begin{array}{c}\text { Image Feature Specification } \\
\text { Queries }\end{array}$ \\
\hline $\begin{array}{c}\text { This compares the given } \\
\text { image to all the images in } \\
\text { database. }\end{array}$ & $\begin{array}{c}\text { This compares feature of } \\
\text { image with pre-collected } \\
\text { features of images. }\end{array}$ \\
\hline $\begin{array}{c}\text { This process is slightly } \\
\text { slower because it inputs } \\
\text { whole image for comparison. }\end{array}$ & $\begin{array}{c}\text { This process is slightly fast as } \\
\text { it inputs only the feature }\end{array}$ \\
\hline
\end{tabular}

Table 2: Similarity Search in Image Data

\begin{tabular}{|c|c|}
\hline $\begin{array}{c}\text { Description-Based } \\
\text { Retrieval Systems }\end{array}$ & $\begin{array}{c}\text { Content-Based Retrieval } \\
\text { Systems }\end{array}$ \\
\hline $\begin{array}{c}\text { This performs object } \\
\text { retrieval based on image } \\
\text { descriptions such as } \\
\text { keywords, captions, size, } \\
\text { and time of creation. }\end{array}$ & $\begin{array}{c}\text { This performs object } \\
\text { retrieval based on content of } \\
\text { the image rather than } \\
\text { metadata of the image. }\end{array}$ \\
$\begin{array}{c}\text { This process is labor } \\
\text { intensive if performed } \\
\text { manually. }\end{array}$ & $\begin{array}{c}\text { This process is automated as } \\
\text { features of the images are } \\
\text { extracted and used for } \\
\text { retrieval. }\end{array}$ \\
\hline $\begin{array}{c}\text { Result of this process is } \\
\text { poor if automated. }\end{array}$ & Results are not affected. \\
\hline
\end{tabular}




\subsection{Interpretation and Evaluation}

Evaluation of retrieval is important in image mining process. Many different methods for measuring the performance of the system have been proposed by researchers. But the most common method for evaluation is Precision and Recall. It is usually presented as precision vs recall graph. The formulae used for evaluating the value for precision and recall are:

$$
\begin{gathered}
\text { Precision }=\frac{\text { no.of relevant images retrived }}{\text { total number of images retrieved }} \\
\text { Recall }=\frac{\text { no.of relevant images retrieved }}{\text { total no.of relevant images in the databse }}
\end{gathered}
$$

\section{LITRATURE REVIEW}

[5] Concerns the extraction of implicit knowledge, image data relationship, or other patterns not explicitly stored in the images. This paper starts with data pre-processing which contains image thresholding, edge detection and border tracing. Extraction of multi-dimensional feature vector such as color, edge, texture is discussed. And finally, learning methodologies (supervised learning and unsupervised learning) followed by association rule mining discussed.

[2] Developed image clustering and categorization technics by using concept of text mining. Applied FUZZY technics on Images and meaningful content. In this approach, user has to first upload the image and give brief description to each image. Then, pre-processing of image starts in which sentence is separated, and divided into verb argument structure. Cluster of the images is created based on frequency of common features among images.

[6] Proposed a novel unsupervised method for the image classification based on various feature distribution of textual images. In first level of classification, image is converted into grayscale image then histogram feature like mean, variance, and skewness are extracted. Using weka-J48, decision tree classifier, images are classified as DOC and NON-DOC images. In second level of classification, grayscale image is sliced in binary form, from that GLCM (grey level coherence matrix) is formed. Then, energy and contrast value of image is calculated. Lastly, depending on these values, decision is taken about image that it is captioned image or scene text image.

[4] Proposed effective clustering to increase speed of image retrieval system. Fuzzy C-means clustering is used to cluster images. Pre-clustering is based on RED group, GREEN group, and BLUE group of images. Entropy is used to compare images with same threshold constraints.

[7] Break the image object into meaningful components such as color, texture, shape, etc. and querying the image objects after representing them to retrieve the discovered knowledge

\subsection{RESEARCH GAP}

$>$ Improvements on image pre-processing technologies, including feature extraction [5].

$>$ Devise highly efficient and extensible image mining algorithms [5].

$>$ Introduce domain knowledge into image mining, which are essential for understanding mining results [5].

$>$ Images are not classified using their own feature instead user interpretation of image is used for clustering images [6].
$>$ Further research should focus on extending the features of image to distinguish images more accurately [8].

$>$ Combination of scene and caption text images, what would be result? [2]

$>$ This application can be used in future to classify the medical images in order to diagnose the right disease verified earlier [4]

$>$ Chosen only most frequent values of some features in order to reduce number of dimensions, which may be the reason of inefficiency [7].

\section{APPLICATION OF IMAGE MINING}

Mining on medical images is to acquire valuable knowledge and modes, which can be used for discovering abnormal situations not consistent with previous modes. Mining can be used to classify CT scan brain images into three categories namely normal, benign, and malign. Image mining can also be used on satellite cloud imagery. Neural network can be used to do image clustering on various image collections. It can assist physicians for efficient classification with multiple keywords per image to improve accuracy.

\section{CONCLUSION}

Image mining is not the newest topic but more work can be done in this area because image objects are hard to define and no best algorithm is proposed till now. Every methodology proposed have issue related to accuracy (input versus output image) or speed of processing. This paper covered various strategies used previously and research gap related to those strategies. These research gaps can be used for implementing a new image mining technique.

\section{REFERENCES}

[1] R. E. W. Rafael C. Gonzalez, DIGITAL IMAGE PROCESSING, PEARSON, 2008.

[2] M. C. D. P. Mr. Dipak R. Pardhi, "Image Classification Using Text Mining and Feature Clustering (Text Document and Image Categorization Using Fuzzy Similarity Based Feature Clustering)," IJETTCS, pp. 136-141, 2015.

[3] K. K. Prabhjeet Kaur, "Review of Different Existing Image Mining Techniques," IJARCSSE, vol. 4, no. 6, pp. 518-524, 2014

[4] D. D. A.Kannan, "Image Clustering and Retrieval using Image Mining Techniques," in IEEE, TAMILNADU, 2010 .

[5] Y. S. HU MIN, "OVERVIEW OF IMAGE MINING RESEARCH," in The 5th International Conference on computer science and education, hefei, china, 2010.

[6] M. J. M. A. M. K. M. B. Prof.Mrs.Sushma Nandgaonkar, "Image Mining of Textual Images Using Low-Leve Image Features," maharastra, IEEE, 2010, pp. 588-592.

[7] A. K. A. S. Imran Khan, "Object Analysis in Image Mining," in INDIAcom, NEW DELHI, 2015.

[8] A. W. Neethu Joseph.c, "Retrieval of images using data mining techniques," KERALA, IEEE, 2014, pp. 204-208. 